\title{
Positive Contrast Imaging of SPIO Nanoparticles
}

\author{
Chenghong Lin, Shuhui Cai, and Jianghua Feng \\ Department of Electronic Science and Fujian Provincial Key Laboratory of Plasma and Magnetic Resonance, Xiamen University, \\ Xiamen 361005, China
}

Correspondence should be addressed to Jianghua Feng, jianghua.feng@xmu.edu.cn

Received 14 December 2011; Revised 19 January 2012; Accepted 27 February 2012

Academic Editor: Carlos Martinez-Boubeta

Copyright ( $) 2012$ Chenghong Lin et al. This is an open access article distributed under the Creative Commons Attribution License, which permits unrestricted use, distribution, and reproduction in any medium, provided the original work is properly cited.

\begin{abstract}
It is advantageous to achieve positive contrast images instead of negative contrast images in superparamagnetic iron-oxide (SPIO) nanoparticles-based MR imaging in order to distinguish the signal surrounding SPIO nanoparticles from the dark signal due to local field inhomogeneity and the artifacts due to tissue interface and background noise, eliminate the inherent defects in the traditional MRI such as partial-volume effects and large void volume for reliable visualization, and increase contrast-to-noise ratio. Many methods generating positive signal with SPIO nanoparticles have been developed in the last decade. This paper provides an overview of current visualization methods and states their advantages and disadvantages. In practice, these techniques have been widely applied to cell labeling and disease diagnosis and monitoring. However, there is still a need for an ideal method to achieve both accuracy and sensitivity.
\end{abstract}

\section{Introduction}

Biocompatible superparamagnetic iron-oxide (SPIO) nanoparticles have been becoming an important class of magnetic resonance imaging (MRI) contrast agents in clinic. The size of these particles ranges from tens to $100 \mathrm{~nm}$, allowing the particles to achieve longer circulation time than other contrast agents such as gadolinium chelates in vivo [1]. These particles consist of iron oxide cores, which are coated with dextran [2] or siloxanes [3] encapsulated by a polymer [4] such as polyethylene glycol (PEG) [5] and further modified to facilitate internalization. Due to their low toxicity and high relaxivity (i.e., lower concentration) in medical practice, they display favorable nanosize-dependent properties beyond gadolinium chelates for MRI diagnosis. However, in the practical use, magnetic heterogeneity is inevitable in the region of interest (ROI) around SPIO nanoparticles due to their superparamagnetic property. Such local field inhomogeneity shortens both $T_{2}^{*}$ and apparent $T_{2}$ relaxation times since spins can relax through different paths, thus giving dark signal in MR images. Meantime, many other factors such as tissue interfaces and background noise can also result in signal loss by inducing local incoherence, which confounds the detection efficiency [6]. Moreover, these negative contrast agents suffer from partial-volume effects, and the void volume for reliable visualization must be larger than voxel size which depends critically on the resolution of the image. Therefore, it is necessary to produce SPIO-specific-positive contrast in order to distinguish the signal surrounding SPIO nanoparticles from the dark signal derived from local field inhomogeneity and reduce the artifacts due to tissue interface and background noise, eliminate the inherent defects in the traditional MRI such as partial-volume effects and large void volume for reliable visualization, and increase contrast-to-noise ratio (CNR).

\section{Magnetic Properties of SPIO Nanoparticles in MRI}

SPIO nanoparticles are sufficiently small so that their magnetizations can randomly flip in direction at room temperature. Similar to paramagnets, they can be magnetized by an external magnetic field. However, they have much larger magnetic susceptibility than paramagnets, thus once the external magnetic field disappears, the magnetism of SPIO nanoparticles will reduce to zero immediately. 
Because SPIO nanoparticles are easily magnetized in a static field and tend to agglomerate, a local magnetic field will be correspondingly produced surrounding SPIO nanoparticles when SPIO nanoparticles are magnetized. The magnetic field is approximately equal to the dipole field from a magnetized sphere $[7,8]$. Moreover, the range of the magnetic field patterns from a collection of SPIO nanoparticles will reduce steeply with the radius of collection. In practice, an agglomeration of SPIO nanoparticles could not be treated as a sphere, but it may be considered as summing patterns from a group of spheres [9].

For the existence of local magnetic heterogeneity, the protons surrounding SPIO nanoparticles are actually in a heterogeneous field with different precession frequency, which leads to faster dephasing. Meanwhile, there are interactions between dipoles of protons and electronic dipoles of SPIO nanoparticles. Accordingly, the $T_{2}$ of protons shortens, which will induce dark signal in the area around SPIO nanoparticles in the conventional MRI.

\section{Positive Contrast Imaging of SPIO Nanoparticles}

In the conventional MRI, SPIO nanoparticles give negative signals that are often confounded by the presence of artifacts due to hemorrhage, air, and partial-volume effects. To address these issues, many attempts have been made to generate positive contrast in the last decade. However, these positivecontrast methods can be classified into three categories as perturbation of magnetic field gradient-based, proton precession frequency-based, and phase (postprocessing)based according to the main theory (see Table 1 for details). It is necessary to have a detailed introduction and evaluation of these methods for a suitable application.

\subsection{Positive Contrast Based on Perturbation of Magnetic Field Gradient}

3.1.1. Gradient Echo Acquisition for Superparamagnetic Particles (GRASP). The GRASP is a typical method for generating positive signal via the perturbation of magnetic field gradient. Originally, Seppenwoolde et al. obtained MR images in which $\mathrm{Dy}_{2} \mathrm{O}_{3}$ marker acted as positive contrast agents [10]. They conserved the signal surrounding the $\mathrm{Dy}_{2} \mathrm{O}_{3}$ marker and dephased the background signal with a slice gradient, and this method was name "white marker". Mani et al. applied this "white marker" to generate positive signal in the presence of SPIO nanoparticles, which was called GRASP [11]. The GRASP sequence is a little different from the conventional gradient echo (GRE) sequence, as shown in Figure 1. It decreases the amplitude of the rephasing gradient (e.g., to $25 \%$ ), which is normally $100 \%$ as slice selection gradient to compensate the slice selection area. The signal of normal circumstances will be effectively weakened due to the gradient imbalance. However, the signal near SPIO nanoparticles will be enhanced, for the gradient balance is restored by added negative local gradient induced by the dipole field of SPIO nanoparticles. The GRASP sequence effectively highlights the regions where SPIO nanoparticles are presented and suppresses the signal of background.

The phantom experiments demonstrated the success of the GRASP sequence in generating positive contrast of SPIO nanoparticles both in 1.5 and $3 \mathrm{~T}$ clinical MRI systems $[11,12]$. Basically, the GRASP sequence can be performed on conventional MRI scanner within short acquisition time, but preliminary experiment is needed to optimize the amplitude of the rephasing gradient. The GRASP sequence is sensitive to field inhomogeneity and background noise is unavoidable. Moreover, it is more effective and sensitive at a moderate field $(1.5 \mathrm{~T})$ than at a higher field $(3 \mathrm{~T})$ due to the higher baseline $R_{2}^{*}$ values at $3 \mathrm{~T}$; therefore the field changes produced by the control cells is actually similar to that from the cells incubated with low concentration of SPIO nanoparticles at $1.5 \mathrm{~T}[11,12]$. High localization and relatively low concentration of SPIO nanoparticles may give optimal enhancement effect [13].

3.1.2. iDQC Anisotropy Map Imaging. In general, intermolecular double-quantum coherences (iDQCs) are sensitive to local magnetic field gradients $[14,15]$. Bouchard et al. obtained three images with the coherence selection gradients oriented in three orthogonal directions $\left(\mathrm{G}_{x}, \mathrm{G}_{y}\right.$, and $\mathrm{G}_{z}$ ), and combined these images to detect the anisotropy in structured samples $[14,16]$. Branca et al. applied this method to generate positive contrast of SPIO nanoparticles called iDQC anisotropy map imaging [6]. The iDQC signal of isotropic media has a spatial dependence $\left(1-3 \cos ^{2} \theta\right)$, where $\theta$ is the angle between the direction of the coherence selection gradient and the external magnetic field $B_{0}$. When the correlation gradient is set along the magic angle $(x+y$ $+z)$, the iDQC signal should be null in the combined image of $\mathrm{G}_{x}+\mathrm{G}_{y}+\mathrm{G}_{z}$ (or $\mathrm{G}_{y}+\mathrm{G}_{x}-\mathrm{G}_{z}$ ) since the image of $\mathrm{G}_{z}$ is twice as strong as the images of $\mathrm{G}_{x}$ and $\mathrm{G}_{y}$. However, the intensity and phase of a structured sample is not zero in the combined image but reflects the local sample structure [16].

The iDQC anisotropy map imaging was proven effective in simulation, phantom, and animal experiments [6]. By using the iDQC imaging techniques, positive signal surrounding SPIO particles can be generated even in a heterogeneous magnetic field, and this technique is insensitive to susceptibility gradients present in most in vivo environments. Therefore it is promising to image the highly heterogeneous areas such as breast tumor and prostate tumor tissues. In addition, the iDQC imaging is feasible for differentiating the focal region (the live part of the tumor) from the background necrotic area in the tumor tissue. It shows exciting possibilities for tumor stage assessment through MRI with specially targeted SPIO nanoparticles [6]. Nevertheless, the major limitation of this method is lower signal-to-noise ratio (SNR) relative to the standard methods. In addition, this method needs a long echo time (TE) for sampling; thus it is unsuitable for tissue with very short $T_{2}$. Promisingly, iDQC imaging could be improved by incorporating new methods for sensitivity enhancement [17-20]. 
TABLE 1: Advantages and disadvantages of the currently used positive contrast imaging methods with SPIO nanoparticles.

\begin{tabular}{|c|c|c|c|c|c|c|c|c|c|}
\hline & \multicolumn{2}{|c|}{$\begin{array}{l}\text { Perturbation of magnetic } \\
\text { field gradient based } \\
\text { methods }\end{array}$} & \multicolumn{4}{|c|}{ Proton precession frequency based methods } & \multicolumn{3}{|c|}{ Phase (postprocessing) based methods } \\
\hline & GRASP & iDQC & SR-SPSP & ORS & IRON & SSFP & PSM & SWI & SGM/PGM \\
\hline $\begin{array}{l}\text { Effective and } \\
\text { sensitive }\end{array}$ & Fair & Low & Fair & Fair & Fair & Fair & High & High & High \\
\hline $\begin{array}{l}\text { Sensitivity to } \\
\text { field } \\
\text { inhomogeneity }\end{array}$ & Yes & No & Yes & Yes & Yes & Yes & Yes & Yes & Yes \\
\hline $\begin{array}{l}\text { Sensitivity to } \\
\text { susceptibility } \\
\text { gradients }\end{array}$ & Yes & No & Yes & Yes & Yes & Yes & Yes & Yes & Yes \\
\hline $\begin{array}{l}\text { Suppression of } \\
\text { background } \\
\text { signal }\end{array}$ & Poor & Good & Fair & Fair & Fair & Fair & Poor & Fair & Fair \\
\hline $\begin{array}{l}\text { Suppression of } \\
\text { water signal }\end{array}$ & Poor & Good & Good & Good & Fair & Good & Poor & Fair & Fair \\
\hline $\begin{array}{l}\text { Signal-to-noise } \\
\text { ratio }\end{array}$ & Fair & Low & High & High & High & High & Fair & Fair & High \\
\hline $\begin{array}{l}\text { Intrinsic } \\
\text { drawback }\end{array}$ & $\begin{array}{l}\text { Low sensitivity } \\
\text { in high field }\end{array}$ & $\begin{array}{l}\text { Require } \\
\text { long TE }\end{array}$ & $\begin{array}{l}\text { Two acqui- } \\
\text { sitions } \\
\text { required }\end{array}$ & $\begin{array}{l}\text { Spurious signals } \\
\text { from field } \\
\text { inhomogeneity }\end{array}$ & & & & & \\
\hline
\end{tabular}

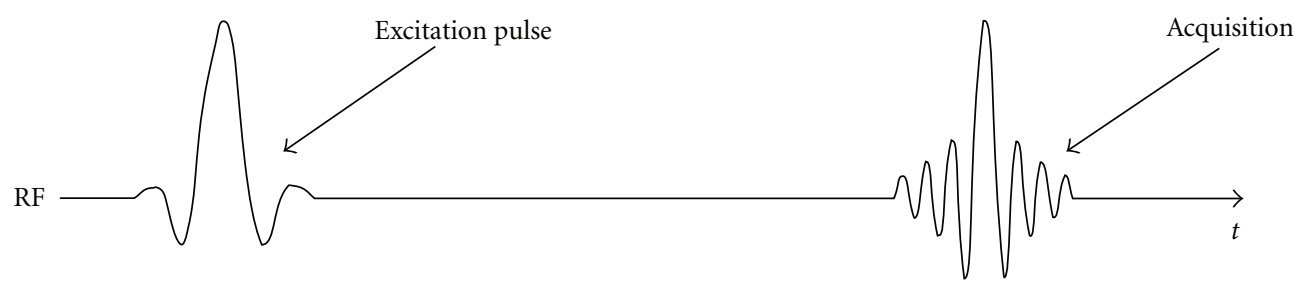

(a)

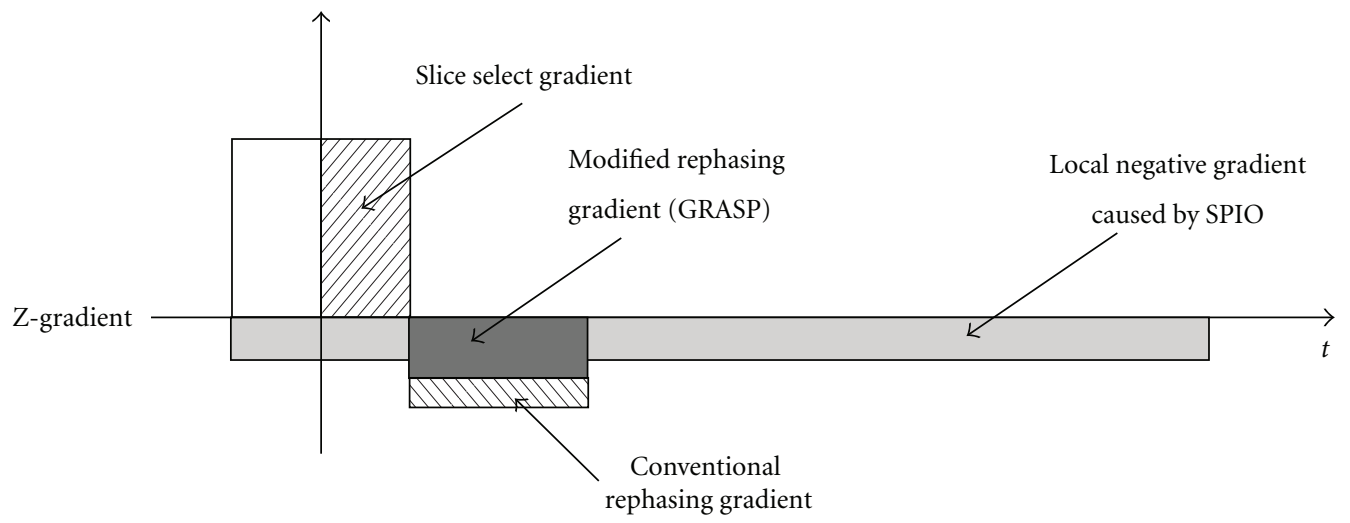

(b)

FIGURE 1: Diagram illustrating the gradients on the slice-select axis for conventional GRE imaging and GRASP sequence for bright imaging of SPIO, regenerated according to the description in [11].

\subsection{Positive Contrast Based on Proton Precession Frequency}

3.2.1. Selective Excitation Imaging. The SPIO nanoparticles will create large magnetization aligned with the static magnetic field, thus inducing an anisotropic resonance frequency distribution with a very well-defined spatial distribution over a short (microns) distance scale surrounding the SPIO nanoparticles [21]. Cunningham et al. used a $90^{\circ}-180^{\circ}$ pulse pair to selectively excite and refocus a narrow band of water molecules [9]. The sequence they used also gave millionfold $(120 \mathrm{~dB})$ suppression of on-resonance water (Figure 2). A frequency selective was used in this sequence [22], and the 


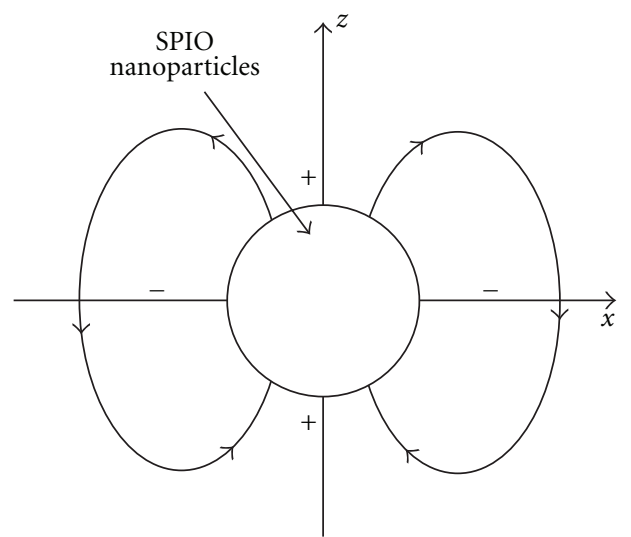

(a)

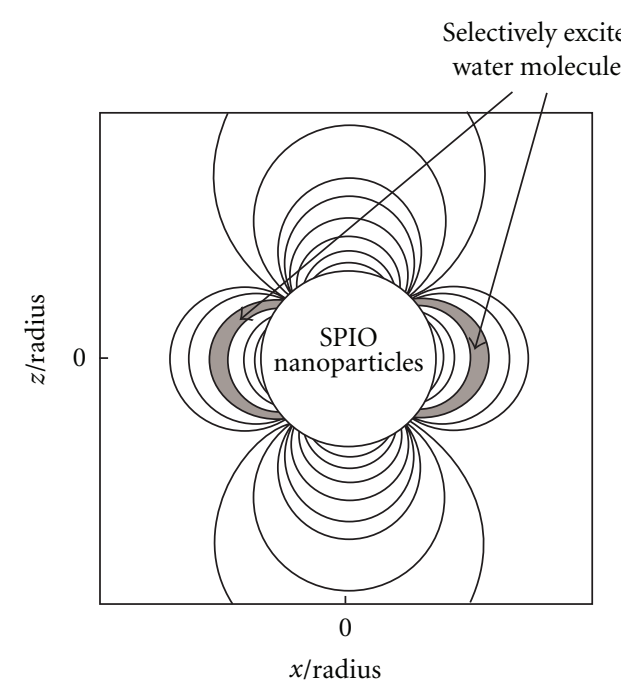

(b)

Figure 2: (a) Magnetic-field lines induced outside a magnetized sphere; (b) water molecules selectively excited by $90^{\circ}-180^{\circ}$ pulse pair, regenerated according to the description in [9].

$90^{\circ}-180^{\circ}$ pair only excites and refocuses a thin shell of spins located at a particular frequency offset surrounding the SPIO nanoparticles [9]. Because the sources of offresonance outside the slice of interest result in interfering signal, Balchandani et al. developed a self-refocused spatialspectral (SR-SPSP) pulse [23], instead of $90^{\circ}-180^{\circ}$ pair, to achieve slice-selective spin-echo imaging of off-resonant spins. The spatial selectivity achieved by the SR-SPSP radio frequency (RF) pulse not only eliminates background signal from regions outside the slice of interest but also reduces the on-resonant water suppression requirement. Such pulse has been tested with phantom and in vivo experiments [23].

The images from phantom and in vivo experiments demonstrated the feasibility of selective excitation imaging on generating positive contrast [9]. Since the sequence never excites on-resonant spins and limits water suppression only in region of interest, water suppression is more efficient $[9,23]$. By changing the transmit frequency and spectral offset of the pulse, it is possible to select flexibly the exciting frequency range with no need to modify the sequence or pulse and gradient waveforms in any static field. However, one limitation of this technique is that two acquisitions are required to eliminate unrefocused components of the magnetization, thus doubling the minimum scan time. In addition, the SR-SPSP pulse lasts $31.8 \mathrm{~ms}$, which leads to another limitation that the minimum achievable repetition time (TR) of the whole pulse is $\sim 43 \mathrm{~ms}$, which is $\sim 20 \mathrm{~ms}$ longer than the TR of the selective excitation imaging used by Cunningham et al. spurious signals from other offresonant sources are still unavoidable [23]. Moreover, its applicability needs to be further investigated due to the employment of high-energy RF pulses that yield increased specific absorption rate (SAR) compared with other methods such as the GRASP sequences.
3.2.2. Off-Resonance Saturation (ORS). Zurkiya and $\mathrm{Hu}$ presented a diffusion-mediated off-resonance saturation method to generate positive contrast of ultrasmall SPIO (USPIO) nanoparticles [24]. A $6 \mathrm{~ms}$ Gaussian pulse was applied in each TR prior to imaging pulses to achieve offresonance irradiation. To quantify the magnitude of the ORS effect, the ORS ratio, $1-\left(\mathrm{M}_{\mathrm{sat}} / \mathrm{M}_{0}\right)$, was calculated, where $M_{\text {sat }}$ was the values of amplitude with off-resonance saturation, and $\mathrm{M}_{0}$ was values of amplitude without offresonance saturation. Since the ORS ratio goes linearly with concentration below saturation concentration, the ORS approach can be used to quantify nanoparticles in an image. This method not only allows assessment of pathophysiological states but also allows reveal of the evolution of disease $[25,26]$.

The ORS demonstrated the favorable feasibility to generate positive signal correlated with USPIO nanoparticle concentration by agarose gel phantom experiments [24, 27]. The ORS effect is highly dependent on diffusion, suggesting that this approach may be useful for the assessment of pathological changes correlating with alteration of the diffusion [28]. However, the use of high-energy RF pulses in this method limits its application due to the increased specific absorption rate.

3.2.3. Inversion-Recovery with On-Resonant Water Suppression (IRON). Stuber et al. developed another technique to visualize the surroundings of SPIO nanoparticles with positive enhancement [29]. As depicted in Figure 3, it was achieved by adding a broadband dual inversion prepulse for fat and on-resonant water suppression to a conventional imaging sequence, thus the signals from fat and background were attenuated significantly. Simultaneously, the area of 


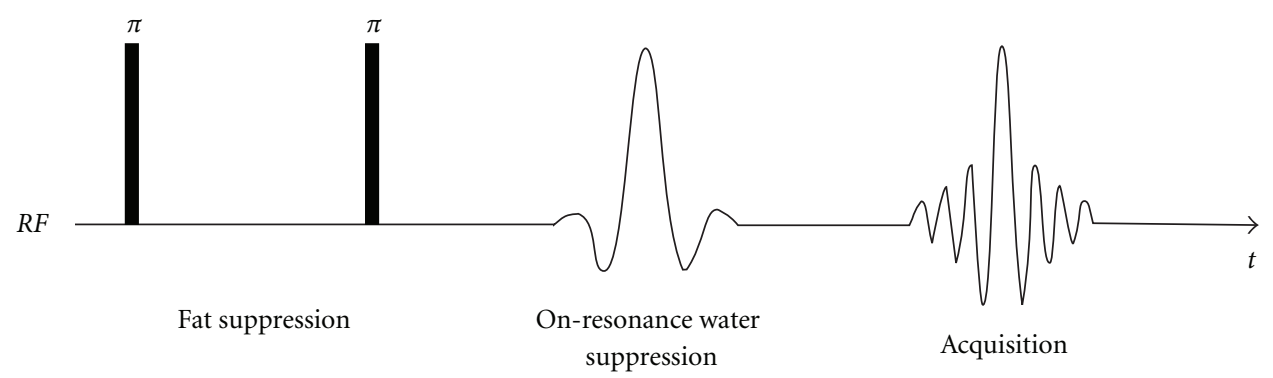

FIGURE 3: Diagram illustrating IRON sequence. Regenerated according to the description in [29].

the positive signal can be controlled by changing the pulse parameters.

IRON imaging facilitates the use of either GRE or fast spin echo (FSE) imaging protocols, for it is unnecessary to modify the imaging part of the sequence [29]. Even so, the prolonged TE may lead a weaker positive signal when IRON is combined with a GRE acquisition. Moreover, when IRON is added to a FSE acquisition, there will be a further increase in absorption rate, especially at higher magnetic field strength. The IRON imaging has been proven to be effective in cylindrical gelatin phantom and in vivo experiments $[12,30]$. The signal of IRON is twice as the signal obtained using selective excitation method because the positive signal is simultaneously contributed from positive and negative frequency components. Additionally, the volume of positive signal shows highly correlated with the concentration of SPIO-labeled cells in vitro [29]. Therefore, IRON may be feasible to quantify the amount of SPIOlabeled cells. However, the volume of positive signal depends on the local concentration and spatial distribution of SPIOlabeled cells which may change quickly in vivo. As a result, the volume of positive signal is not exactly correlated with the number of SPIO-labeled cells in vivo. Moreover, as one of the sequences that use signal dephasing or off-resonance as a way of selecting SPIO signal, spurious positive signal could be unavoidable at tissue borders or air-tissue interfaces during IRON imaging, and residual background noise due to $B_{1}$ inhomogeneity could also lead to local "over/under tipping" of the magnetization [29]. Furthermore, because the time of $T_{1}$ recovery during the RF excitation train suffers from the dual inversion for fat suppression, the broadband dual inversion needs to be replaced with another spectrally selective prepulse for fat saturation to achieve more effective on-resonant water suppression. Finally, the threshold of detectability of SPIO-labeled cells is not clear yet for IRON imaging.

3.2.4. Balanced Steady-State Free Precession. Alternative dual inversion prepulse and on-resonant water suppression pulse was proposed by Dharmakumar et al. to generate positive contrast from off-resonant spins with steady-state free precession (SSFP) magnetic resonance imaging [31]. On that basis, Mascheri et al. got positive contrast imaging of USPIO-labeled macrophages with a low-tip-angle balanced steady-state free precession (bSSFP) sequence at clinical field strength $(1.5 \mathrm{~T})$ and resolution $\left(0.8 \times 0.8 \times 3 \mathrm{~mm}^{3}\right)$ [32]. Further, Çukur et al. presented an improved method, positive contrast with alternating repetition time steady-state free precession (PARTS) [33], which coupled low tip angles for generating off-resonant signal with alternating repetition times $[34,35]$ for water suppression. This sequence is combined with a separate acquisition where the stopband is instead centered at the fat resonance.

Both PARTS and bSSFP shows effective in phantom and in vivo experiments $[32,33,35,36]$. PARTS improved background suppression, thus allowing quantitative positive contrast measurements in vitro [33]. However, absolute quantification in vivo may be impossible due to the unexpectable processes such as the migration, clustering, proliferation, or death of cells. Meanwhile, PARTS is difficult to fit with higher field strength unless giving shorter TRs. Spurious signals from considerable field inhomogeneity and susceptibility boundaries are inescapable in PARTS images as other off-resonance techniques, which limit the specificity of positive contrast images with PARTS around the SPIOlabeled cells. Based on a low-tip-angle alternating repetition time (ATR) SSFP sequence, PARTS has higher SNR, lower specific absorption rate, and lower flow sensitivity than other off-resonance methods. The acquisition time of PARTS is extraordinarily short, even four to five times shorter than GRE sequences. Furthermore, PARTS can produce threedimensional high-resolution images with positive contrast within less than one minute. The smallest number of cells detectable with the method ( 0.1 million in vitro) is not inferior to other methods $[9,29]$, but the detectability thresholds are yet to be determined.

\subsection{Positive Contrast Based on Phase}

3.3.1. Phase Slope Magnitude (PSM) Imaging. Exploiting the characteristic of phase or susceptibility is another feasible approach to generate positive signal of sites surrounding SPIO nanoparticles, which is one of postprocessing methods. The PSM image applied a high-pass filtering to the phase images [37, 38], which preserved image resolution and improved quantification sensitivity simultaneously.

The PSM imaging was proven to be effective in gel phantom and mouse experiments $[37,38]$. Extraordinarily, the 
images exhibit a 1 - to 8 -fold CNR improvement in regions containing the SPIO deposits. Moreover, it benefits from the increased sensitivity to magnetic susceptibility effects while retaining the critical anatomic backdrop by superimposing PSM images onto magnitude images. Unlike many other positive contrast imaging methods, PSM imaging does not require any modification to acquisition, and it can be combined with any imaging sequence. However, there are several inherent drawbacks for the PSM method. Firstly, the spurious signals which derived from some tissue interfaces will be unavoidable. Secondly, computational artifacts caused by rapid spatial changes in phase angle are possibly introduced into PSM images under the existence of highly relaxative SPIO nanoparticles [25]. If image resolution is too low to sample the rapid phase changes induced by highly relaxative SPIO nanoparticles in phase angle, undersampling will occur, which can potentially cause phase unwrapping problem, thus leads to an additional phase to each phase image pixel with multiple of $2 \pi$. Finally, as a postprocessings method, the PSM images are more sensitive to motion artifacts than those mentioned above [39].

3.3.2. Variation of Susceptibility-Weighted Imaging (SWI). Eibofner et al. proposed a positive contrast technique combining the magnitude image with phase image of a gradient-echo dataset [40]. It is a variation of SWI technique developed by Haacke et al. [41]. A mask generated from the values of phase is multiplied by the values of amplitude, resulting in the susceptibility weighted image. By changing the properties of the phase mask, the sites surrounding SPIO nanoparticles can be highlighted against the homogeneous sites. Three different masks defined zero on phase values, Hanning mask, V-shaped mask, and step function mask were implemented in order to suppress the on-resonant spins which were defined on a certain phase shift to keep signals from protons surrounding SPIO nanoparticles.

The SWI method for SPIO nanoparticles showed good potential for positive contrast imaging on agar phantom and ex vivo bovine liver experiment [40]. This method can be regarded as a simple version of PSM imaging [40, 42]. Moreover, this postprocessing is insensitive toward an inaccurate shimming or inhomogeneous magnetic field because the phase filter procedure removes the low spatial frequency components. Another advantage of this technique is that the negative and the positive dipole distortions contribute positive signal simultaneously, which is accomplished by the symmetrical phase mask. However, the accuracy of this technique is limited by the inhomogeneities of the tissues around the SPIO nanoparticles and the inhomogeneity of the magnetic field, even the excitation profile of the coil. In inhomogeneous tissues such as bovine liver, due to a complete suppression of the background signal, the smallest number of SPIO-labeled cells detected significantly increases to 23,000 while concentration of 1000 cells $/ 20 \mathrm{~mL}$ could be visualized in agar phantom [40]. Furthermore, this method can only be applied to the gradient echo sequence, in which lower readout bandwidth (BW) and longer TE would be better.
3.3.3. Susceptibility Gradient Mapping (SGM) and Phase Gradient Mapping (PGM). Dahnke et al. presented another postprocessing method called SGM [42], which was based on the fact that susceptibility-induced field gradients could be visualized after applying Fourier transform on image subsets [43]. Zhao et al. introduced an improved method PGM in which a fast Fourier transform (FFT) approach was implemented and no phase unwrapping procedure was required [44]. The PGM method generates effective positive contrast maps coming from the susceptibility gradients associated with SPIO particles. The PGM method generates positive signal with sensitivity higher than that of SGM at medium and low concentrations of SPIO, while SGM is more sensitive than PGM at longer TEs.

Both PGM and SGM show accurate estimation on the SPIO concentration from phantom dataset [12, 42, 44]. These postprocessing methods offer additional information from a $T_{2}^{*}$-weighted image without any extra measurement. Moreover, they can be used to discriminate hypointensities induced by susceptibility gradients from others though the provided susceptibility gradient map has a lower resolution than the original image. PGM and SGM show a great potential to detect the susceptibility induced by contrast agents or medical devices [45]. However, the unavoidable spurious signal from some tissue interfaces and the detected threshold of cells from inhomogeneous tissues are still a concern practical application.

\section{Application of Positive Contrast Imaging with SPIO Nanoparticles}

The traditional MR image could be significantly improved by positive contrast imaging of SPIO nanoparticles as contrast agents, and that has stimulated an increasing number of new techniques. Their applications have been expanded to the field of disease diagnosis and monitoring even clinical use [32], with tracking transplanted cells in various organs being the most widely investigation [46-48].

Cunningham et al. [9] and Balchandani et al. [23] achieved frequency-selective excitation positive signal of SPIO-labeled cells, in which the cells were injected into the hind limbs of a living mouse. Stuber et al. obtained threedimensional IRON FSE images of rabbit hind limbs with 250,000 and 125,000 SPIO-labeled stem cells, respectively [29]. Both injection sites can be readily detected as areas of hyperintense signal at $3 \mathrm{~T}$. Suzuki et al. compared offresonance (OR) positive contrast with GRE acquisition of SPIO-luc-mESC (mouse embryonic stem cell transfected with luciferase reporter gene) transplanted to mice and calculated the SNR and CNR obtained from OR and GRE acquisition. Both SNR and CNR from OR acquisition are significantly higher than those from GRE acquisition [49]. Cukur et al. tracked SPIO-labeled human bone marrow stromal cells injected into mice with PARTS sequence [33]. No longer satisfied with locating the site of cells, Brisset et al. quantified cells with GRASP sequence in mouse brains [50]. They found that the measurement of cloverleaf artifact volume was significantly related to the given range 
of cells. Zhao et al. used PGM and SGM images to quantify SPIO-labeled cells which were injected subcutaneously and bilaterally into the flanks of rats [44]. Besides tracking cells, positive contrast imaging with SPIO nanoparticles shows effective in detection of burn infection [51], atherosclerosis $[52,53]$, crush injury [13], and so on.

Although positive contrast imaging with SPIO nanoparticles solves some problems in conventional MRI, such as differentiation of SPIO nanoparticles and other noise, some problems still need further investigate, such as accuracy of detection, dependability of cells tracking with long-term, spurious signal, and elimination of susceptibility boundaries.

Besides, the research of multifunctional materials is very active in last years. Some multifunctional SPIO materials have been produced as $T_{2}$-agent in MRI, biological indicators, rhodamine $\mathrm{B}$ isothiocyanate, $\mathrm{T}_{1}$-agent (dual $\mathrm{MR}$ contrast), and antibacterials [54-59]. These materials will bring many fresh applications of positive contrast imaging with SPIO.

\section{Conclusions and Future Work}

It is advantageous to achieve positive contrast images of SPIOs. An ideal SPIO-based positive-contrast imaging technique ought to (1) have high sensitivity and good specificity, (2) have insensitivity for field inhomogeneity and susceptibility gradients not associated with SPIOs, (3) have good suppression capability for background and onresonant water signals, and (4) have high signal-to-noise ratio. In reality, no method will meet all these requirements as shown in Table 1 but a good positive-contrast method ideally should meet as many criteria as possible in the specific application. In practice, positive contrast imaging with SPIO nanoparticles has been successfully applied to tracking cells and disease diagnosis and monitoring. In all currently used methods, every method has its advantages, but a better method is expected to detect the SPIO nanoparticles with improved accuracy and sensitivity. It is clear that the best choice would be to take advantages of the combination of some existing methods. It is predictable that such integrate method will become increasing important and facilitate the clinical application in the future.

\section{Acknowledgments}

This work was financially supported by the NNSF of China (11074209), Fundamental Research Funds for the Central Universities (2011121046), and Xiamen Research Platform on Systems Biology of Metabolic Disease.

\section{References}

[1] C. Boyer, M. R. Whittaker, V. Bulmus, J. Liu, and T. P. Davis, "The design and utility of polymer-stabilized iron oxide nanoparticles for nanomedicine applications," NPG Asia Materials, vol. 2, no. 1, pp. 23-30, 2010.

[2] T. Shen, R. Weissleder, M. Papisov, A. Bogdanov, and T. J. Brady, "Monocrystalline iron oxide nanocompounds
(MION): physicochemical properties," Magnetic Resonance in Medicine, vol. 29, no. 5, pp. 599-604, 1993.

[3] C. W. Jung and P. Jacobs, "Physical and chemical properties of superparamagnetic iron oxide MR contrast agents: ferumoxides, ferumoxtran, ferumoxsil," Magnetic Resonance Imaging, vol. 13, no. 5, pp. 661-674, 1995.

[4] K. A. Hinds, J. M. Hill, E. M. Shapiro et al., "Highly efficient endosomal labeling of progenitor and stem cells with large magnetic particles allows magnetic resonance imaging of single cells," Blood, vol. 102, no. 3, pp. 867-872, 2003.

[5] M. Mahmoudi, A. Simchi, M. Imani, and U. O. Hafeli, "Superparamagnetic iron oxide nanoparticles with rigid crosslinked polyethylene glycol fumarate coating for application in imaging and drug delivery," Journal of Physical Chemistry C, vol. 113, no. 19, pp. 8124-8131, 2009.

[6] R. T. Branca, Y. M. Chen, V. Mouraviev et al., "IDQC anisotropy map imaging for tumor tissue characterization in vivo," Magnetic Resonance in Medicine, vol. 61, no. 4, pp. 937943, 2009

[7] D. A. Yablonskiy and E. M. Haacke, "Theory of NMR signal behavior in magnetically inhomogeneous tissues: the static dephasing regime," Magnetic Resonance in Medicine, vol. 32, no. 6, pp. 749-763, 1994.

[8] J. F. Schenck, "The role of magnetic susceptibility in magnetic resonance imaging: MRI magnetic compatibility of the first and second kinds," Medical Physics, vol. 23, no. 6, pp. 815-850, 1996.

[9] C. H. Cunningham, T. Arai, P. C. Yang, M. V. McConnell, J. M. Pauly, and S. M. Conolly, "Positive contrast magnetic resonance imaging of cells labeled with magnetic nanoparticles," Magnetic Resonance in Medicine, vol. 53, no. 5, pp. 999-1005, 2005.

[10] J. H. Seppenwoolde, M. A. Viergever, and C. J. G. Bakker, "Passive tracking exploiting local signal conservation: the white marker phenomenon," Magnetic Resonance in Medicine, vol. 50, no. 4, pp. 784-790, 2003.

[11] V. Mani, K. C. Briley-Saebo, V. V. Itskovich, D. D. Samber, and Z. A. Fayad, "Gradient echo acquisition for superparamagnetic particles with positive contrast (GRASP): sequence characterization in membrane and glass superparamagnetic iron oxide phantoms at 1.5T and 3T," Magnetic Resonance in Medicine, vol. 55, no. 1, pp. 126-135, 2006.

[12] W. Liu, H. Dahnke, E. K. Jordan, T. Schaeffter, and J. A. Frank, "In vivo MRI using positive-contrast techniques in detection of cells labeled with superparamagnetic iron oxide nanoparticles," NMR in Biomedicine, vol. 21, no. 3, pp. 242250, 2008.

[13] V. Mani, K. C. Briley-Saebo, F. Hyafil, and Z. A. Fayad, "Feasibility of in vivo identification of endogenous ferritin with positive contrast MRI in rabbit carotid crush injury using GRASP," Magnetic Resonance in Medicine, vol. 56, no. 5, pp. 1096-1106, 2006.

[14] L. S. Bouchard, R. R. Rizi, and W. S. Warren, "Magnetization structure contrast based on intermolecular multiple-quantum coherences," Magnetic Resonance in Medicine, vol. 48, no. 6, pp. 973-979, 2002.

[15] Z. Chen, X. Zhu, B. Zheng, S. Cai, and J. Zhong, "Doublequantum-filtered intermolecular single-quantum coherences in nuclear magnetic resonance spectroscopy and imaging," Chemical Physics Letters, vol. 429, no. 4-6, pp. 611-616, 2006.

[16] L. S. Bouchard and W. S. Warren, "Multiple-quantum vector field imaging by magnetic resonance," Journal of Magnetic Resonance, vol. 177, no. 1, pp. 9-21, 2005. 
[17] C. Cai, F. Gao, S. Cai, J. Zhong, and Z. Chen, "Highly efficient square wave distant dipolar field and its application for in vivo MRI," Magnetic Resonance in Medicine, vol. 64, no. 4, pp. 1128-1134, 2010.

[18] R. T. Branca, G. Galiana, and W. S. Warren, "Enhanced nonlinear magnetic resonance signals via square wave dipolar fields," Journal of Chemical Physics, vol. 129, no. 5, Article ID 054502, 2008.

[19] R. T. Branca, G. Galiana, and W. S. Warren, "Signal enhancement in CRAZED experiments," Journal of Magnetic Resonance, vol. 187, no. 1, pp. 38-43, 2007.

[20] T. Hou, Z. Chen, D. W. Hwang, J. H. Zhong, and L. P. Hwang, "Intermolecular double-quantum coherence MR microimaging of pig tail with unique image contrast," Magnetic Resonance Imaging, vol. 22, no. 4, pp. 543-550, 2004.

[21] T. Enss, S. Ahn, and W. S. Warren, "Visualizing the dipolar field in solution NMR and MR imaging: three-dimensional structure simulations," Chemical Physics Letters, vol. 305, no. 1-2, pp. 101-108, 1999.

[22] A. N. Garroway, P. K. Grannell, and P. Mansfield, "Image formation in NMR by a selective irradiative process," Journal of Physics C, vol. 7, no. 24, article no. 006, pp. L457-L462, 1974.

[23] P. Balchandani, M. Yamada, J. Pauly, P. Yang, and D. Spielman, "Self-refocused spatial-spectral pulse for positive contrast imaging of cells labeled with SPIO nanoparticles," Magnetic Resonance in Medicine, vol. 62, no. 1, pp. 183-192, 2009.

[24] O. Zurkiya and X. Hu, "Off-resonance saturation as a means of generating contrast with superparamagnetic nanoparticles," Magnetic Resonance in Medicine, vol. 56, no. 4, pp. 726-732, 2006.

[25] C. Zimmer, S. C. Wright, R. T. Engelhardt et al., "Tumor cell endocytosis imaging facilitates delineation of the glioma-brain interface," Experimental Neurology, vol. 143, no. 1, pp. 61-69, 1997.

[26] H. C. Roberts, T. P. L. Roberts, S. Ley, W. P. Dillon, and R. C. Brasch, "Quantitative estimation of microvascular permeability in human brain tumors: correlation of dynamic GdDTPA-enhanced MR imaging with histopathologic grading," Academic Radiology, vol. 9, no. 1, supplement, pp. S151-S155, 2002.

[27] C. Khemtong, O. Togao, J. Ren et al., "Off-resonance saturation MRI of superparamagnetic nanoprobes: theoretical models and experimental validations," Journal of Magnetic Resonance, vol. 209, no. 1, pp. 53-60, 2011.

[28] P. Krämer, X. Helluy, T. Kampf, E. Lang, and P. M. Jakob, "Flow-enhanced off-resonance saturation for remote detection of iron-based contrast agents," Magnetic Resonance in Medicine, vol. 63, no. 6, pp. 1708-1715, 2010.

[29] M. Stuber, W. D. Gilson, M. Schär et al., "Positive contrast visualization of iron oxide-labeled stem cells using inversionrecovery with ON-resonant water suppression (IRON)," Magnetic Resonance in Medicine, vol. 58, no. 5, pp. 1072-1077, 2007.

[30] G. Korosoglou, L. Tang, D. Kedziorek et al., "Positive contrast MR-lymphography using inversion recovery with ONresonant water suppression (IRON)," Journal of Magnetic Resonance Imaging, vol. 27, no. 5, pp. 1175-1180, 2008.

[31] R. Dharmakumar, I. Koktzoglou, and D. Li, "Generating positive contrast from off-resonant spins with steady-state free precession magnetic resonance imaging: theory and proof-ofprinciple experiments," Physics in Medicine and Biology, vol. 51, no. 17, article no. 006, pp. 4201-4215, 2006.
[32] N. Mascheri, R. Dharmakumar, Z. Zhang, T. Paunesku, G. Woloschak, and D. Li, "Fast low-angle positive contrast steadystate free precession imaging of USPIO-labeled macrophages: theory and in vitro experiment," Magnetic Resonance Imaging, vol. 27, no. 7, pp. 961-969, 2009.

[33] T. Çukur, M. Yamada, W. R. Overall, P. Yang, and D. G. Nishimura, "Positive contrast with alternating repetition time SSFP (PARTS): a fast imaging technique for SPIO-labeled cells," Magnetic Resonance in Medicine, vol. 63, no. 2, pp. 427437, 2010.

[34] J. Leupold, J. Hennig, and K. Scheffler, "Alternating repetition time balanced steady state free precession," Magnetic Resonance in Medicine, vol. 55, no. 3, pp. 557-565, 2006.

[35] K. S. Nayak, H. L. Lee, B. A. Hargreaves, and B. S. Hu, "Wideband SSFP: alternating repetition time balanced steady state free precession with increased band spacing," Magnetic Resonance in Medicine, vol. 58, no. 5, pp. 931-938, 2007.

[36] S. Patil, D. Jirák, F. Saudek, M. Hájek, and K. Scheffler, "Positive contrast visualization of SPIO-labeled pancreatic islets using echo-dephased steady-state free precession," European Radiology, vol. 21, no. 1, pp. 214-220, 2011.

[37] P. H. Mills, Y. J. L. Wu, C. Ho, and E. T. Ahrens, "Sensitive and automated detection of iron-oxide-labeled cells using phase image cross-correlation analysis," Magnetic Resonance Imaging, vol. 26, no. 5, pp. 618-628, 2008.

[38] P. H. Mills and E. T. Ahrens, "Enhanced positive-contrast visualization of paramagnetic contrast agents using phase images," Magnetic Resonance in Medicine, vol. 62, no. 5, pp. 1349-1355, 2009.

[39] A. Rauscher, M. Barth, J. R. Reichenbach, R. Stollberger, and E. Moser, "Automated unwrapping of MR phase images applied to BOLD MR-venography at 3 Tesla," Journal of Magnetic Resonance Imaging, vol. 18, no. 2, pp. 175-180, 2003.

[40] F. Eibofner, G. Steidle, R. Kehlbach, R. Bantleon, and F. Schick, "Positive contrast imaging of iron oxide nanoparticles with susceptibility-weighted imaging," Magnetic Resonance in Medicine, vol. 64, no. 4, pp. 1027-1038, 2010.

[41] E. M. Haacke, Y. Xu, Y. C. N. Cheng, and J. R. Reichenbach, "Susceptibility weighted imaging (SWI)," Magnetic Resonance in Medicine, vol. 52, no. 3, pp. 612-618, 2004.

[42] H. Dahnke, W. Liu, D. Herzka, J. A. Frank, and T. Schaeffter, "Susceptibility gradient mapping (SGM): a new postprocessing method for positive contrast generation applied to superparamagnetic iron oxide particle (SPIO)-labeled cells," Magnetic Resonance in Medicine, vol. 60, no. 3, pp. 595-603, 2008.

[43] J. R. Reichenbach, R. Venkatesan, D. A. Yablonskiy, M. R. Thompson, S. Lai, and E. M. Haacke, "Theory and application of static field inhomogeneity effects in gradientecho imaging," Journal of Magnetic Resonance Imaging, vol. 7, no. 2, pp. 266-279, 1997.

[44] Q. Zhao, J. Langley, S. Lee, and W. Liu, "Positive contrast technique for the detection and quantification of superparamagnetic iron oxide nanoparticles in MRI," NMR in Biomedicine, vol. 24, no. 5, pp. 464-472, 2011.

[45] H. Zhu, K. Demachi, and M. Sekino, "Phase gradient imaging for positive contrast generation to superparamagnetic iron oxide nanoparticle-labeled targets in magnetic resonance imaging," Magnetic Resonance Imaging, vol. 29, no. 7, pp. 891$898,2011$.

[46] A. A. Gilad, P. Walczak, M. T. McMahon et al., "MR tracking of transplanted cells with "positive contrast" using manganese oxide nanoparticles," Magnetic Resonance in Medicine, vol. 60, no. 1, pp. 1-7, 2008. 
[47] D. Li, Z. Zhang, R. Dharmakumar, N. Mascheri, Z. Fan, and $\mathrm{S}$. Wu, "Comparison of superparamagnetic and ultrasmall superparamagnetic iron oxide cell labeling for tracking green fluorescent protein gene marker with negative and positive contrast magnetic resonance imaging," Molecular Imaging, vol. 8, no. 3, pp. 148-155, 2009.

[48] J. Chung, K. Kee, J. K. Barral et al., "In vivo molecular MRI of cell survival and teratoma formation following embryonic stem cell transplantation into the injured murine myocardium," Magnetic Resonance in Medicine, vol. 66, no. 5, pp. 1347-1381, 2011.

[49] Y. Suzuki, C. H. Cunningham, K. I. Noguchi et al., "In vivo serial evaluation of superparamagnetic iron-oxide labeled stem cells by off-resonance positive contrast," Magnetic Resonance in Medicine, vol. 60, no. 6, pp. 1269-1275, 2008.

[50] J. C. Brisset, M. Sigovan, F. Chauveau et al., "Quantification of iron-labeled cells with positive contrast in mouse brains," Molecular Imaging and Biology, pp. 1-7, 2010.

[51] O. C. Andronesi, D. Mintzopoulos, N. Psychogios et al., "Combined off-resonance imaging and T2 relaxation in the rotating frame for positive contrast MR imaging of infection in a murine burn model," Journal of Magnetic Resonance Imaging, vol. 32, no. 5, pp. 1172-1183, 2010.

[52] G. Korosoglou, R. G. Weiss, D. A. Kedziorek et al., "Noninvasive detection of macrophage-rich atherosclerotic plaque in hyperlipidemic rabbits using "positive contrast" magnetic resonance imaging," Journal of the American College of Cardiology, vol. 52, no. 6, pp. 483-491, 2008.

[53] K. C. Briley-Saebo, V. Mani, F. Hyafil, J. C. Cornily, and Z. A. Fayad, "Fractionated Feridex and positive contrast: in vivo MR imaging of atherosclerosis," Magnetic Resonance in Medicine, vol. 59, no. 4, pp. 721-730, 2008.

[54] G. Zabow, S. Dodd, J. Moreland, and A. Koretsky, "Microengineered local field control for high-sensitivity multispectral MRI," Nature, vol. 453, no. 7198, pp. 1058-1063, 2008.

[55] T. H. Kim, J. K. Kim, W. Shim, S. Y. Kim, T. J. Park, and J. Y. Jung, "Tracking of transplanted mesenchymal stem cells labeled with fluorescent magnetic nanoparticle in liver cirrhosis rat model with 3-T MRI," Magnetic Resonance Imaging, vol. 28, no. 7, pp. 1004-1013, 2010.

[56] Y. Onuki, I. Jacobs, D. Artemov, and Y. Kato, "Noninvasive visualization of in vivo release and intratumoral distribution of surrogate MR contrast agent using the dual MR contrast technique," Biomaterials, vol. 31, no. 27, pp. 7132-7138, 2010.

[57] Z. Wei, Z. Zhou, M. Yang et al., "Multifunctional Ag@ $\mathrm{Fe}_{2} \mathrm{O}_{3}$ yolk-shell nanoparticles for simultaneous capture, kill, and removal of pathogen," Journal of Materials Chemistry, vol. 21, no. 41, Article ID 16344, 2011.

[58] H. Yang, Y. Zhuang, Y. Sun et al., "Targeted dual-contrast $T_{1}$ and $T_{2}$-weighted magnetic resonance imaging of tumors using multifunctional gadolinium-labeled superparamagnetic iron oxide nanoparticles," Biomaterials, vol. 32, no. 20, pp. 45844593, 2011.

[59] F. Wang, X. Chen, Z. Zhao et al., "Synthesis of magnetic, fluorescent and mesoporous core-shell-structured nanoparticles for imaging, targeting and photodynamic therapy," Journal of Materials Chemistry, vol. 21, no. 30, pp. 11244-11252, 2011. 

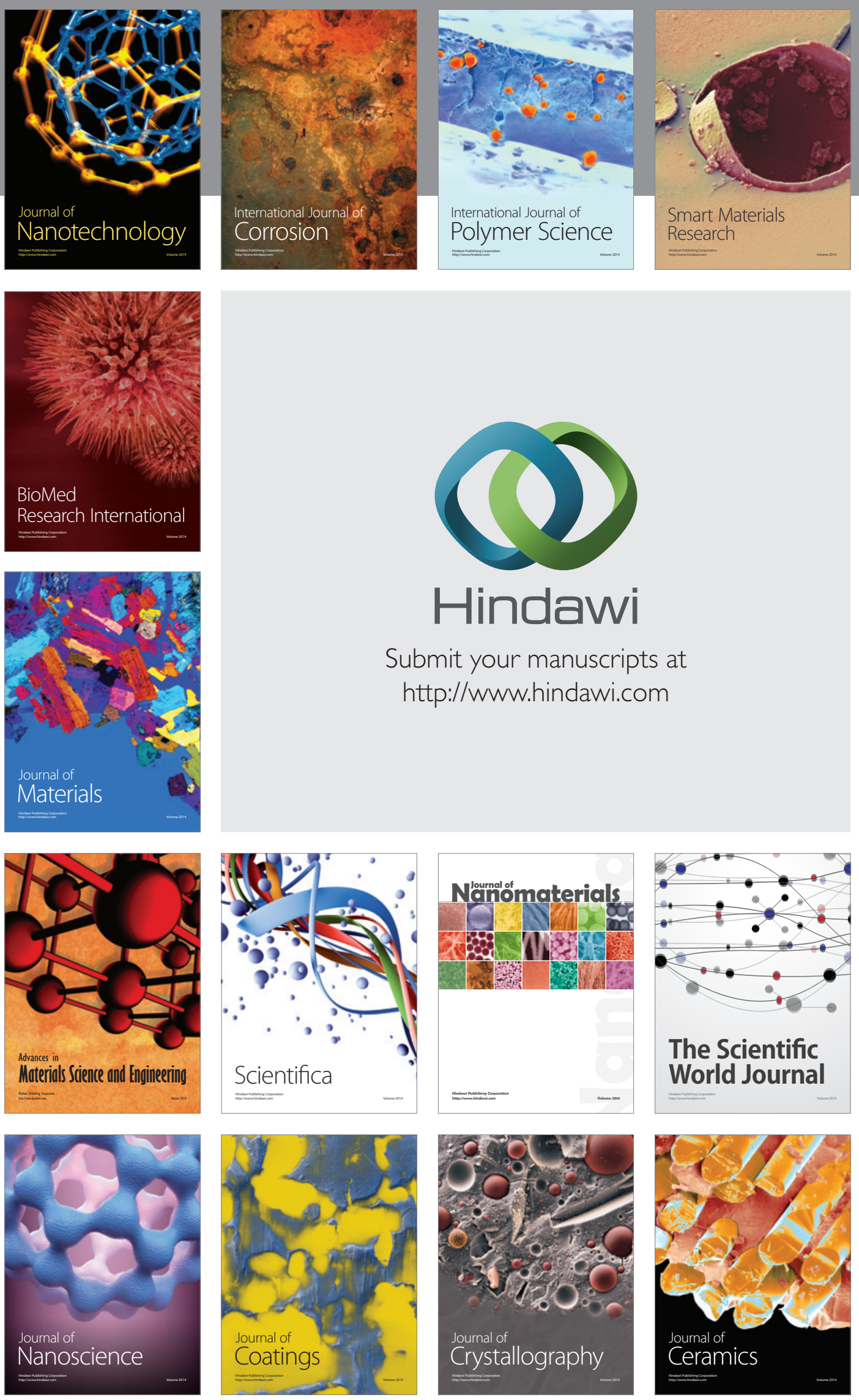

The Scientific World Journal

Submit your manuscripts at

http://www.hindawi.com

\section{World Journal}

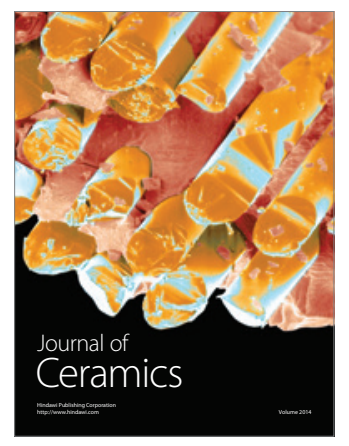

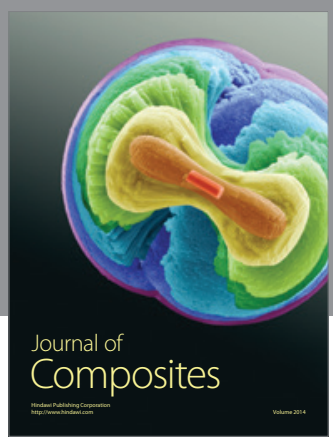
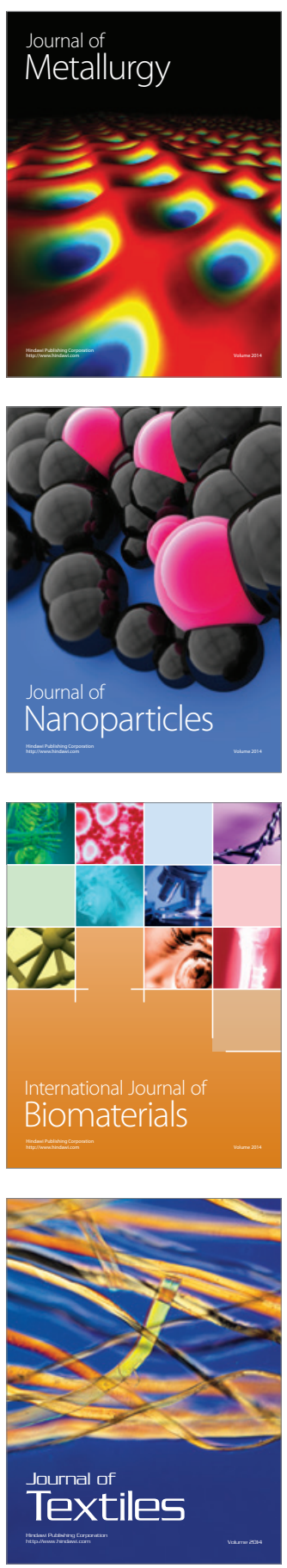